University of Nebraska - Lincoln

DigitalCommons@University of Nebraska - Lincoln

$5-1-2021$

\title{
The Experts Speak: A New Feature in the JICR
}

\author{
Michael Gale \\ University of Washington School of Medicine \\ Ram Savan \\ University of Washington School of Medicine \\ Adolfo Garcia-Sastre \\ Icahn School of Medicine at Mount Sinai \\ Ludmila Prokunina-Olsso \\ National Cancer Institute (NCl) \\ Kuppusamy Balamurugan \\ National Cancer Institute at Frederick
}

See next page for additional authors

Follow this and additional works at: https://digitalcommons.unl.edu/publichealthresources

Part of the Medicine and Health Sciences Commons

Gale, Michael; Savan, Ram; Garcia-Sastre, Adolfo; Prokunina-Olsso, Ludmila; Balamurugan, Kuppusamy; and Young, Howard A., "The Experts Speak: A New Feature in the JICR" (2021). Public Health Resources. 566.

https://digitalcommons.unl.edu/publichealthresources/566

This Article is brought to you for free and open access by the Public Health Resources at DigitalCommons@University of Nebraska - Lincoln. It has been accepted for inclusion in Public Health Resources by an authorized administrator of DigitalCommons@University of Nebraska - Lincoln. 


\section{Authors}

Michael Gale, Ram Savan, Adolfo Garcia-Sastre, Ludmila Prokunina-Olsso, Kuppusamy Balamurugan, and Howard A. Young 


\title{
The Experts Speak:
}

\section{A New Feature in the JICR}

\author{
Michael Gale, Jr, Ram Savan, ${ }^{1}$ Adolfo Garcia-Sastre, ${ }^{2}$ Ludmila Prokunina-Olsson, ${ }^{3}$ \\ Kuppusamy Balamurugan, ${ }^{4}$ and Howard A. Young ${ }^{4}$
}

This month we launch a new feature in the JICR: The Experts Speak. This feature is designed to provide first-hand insight from topical experts in the fields of interferon, cytokines, innate immunity, and adaptive immune actions of cytokine biology in disease. We start by asking experts to comment on interferon, cytokines, and SARS-CoV-2.

Michael Gale, Jr. Editor-in-Chief

JICR

\section{Interferon and Innate Immunity Can Control SARS-CoV-2 Infection, but How Do They Work?}

At this point in the SARS-CoV-2 pandemic, we have learned that approximately $75-85 \%$ of people infected with the virus develop either an asymptomatic infection or mild to moderate COVID-19 (the disease linked with SARSCoV-2 infection) with recovery, while it is a minority of infected people who develop severe disease or disease leading to death. However, the sheer number of people infected with SARS-CoV-2 locally and world-wide underscore the severity of the pandemic. Moreover, "COVID-19 long haulers" who display varied disease symptoms long after viral clearance present a growing concern to the continued public health impact of SARS-CoV-2. Types I and III interferon (IFN) are essential for defense against virus infection, and they can clearly impart suppression of SARS$\mathrm{CoV}-2$ infection when administered to cultured cells prior to virus infection. SARS-CoV-2 encodes multiple proteins than can antagonize innate immune programs of the infected cell, including proteins that suppress IFN signaling and effector actions such that when administered after infection IFN is not effective to suppress viral replication. When clinically administered as a therapeutic to infected hospitalized patients, it is clear that early treatment with IFN can be beneficial and reduce inpatient time to recovery. IFN actions are mediated by interferon-stimulated genes (ISGs), the main effectors that directly suppress virus replication and/or serve to regulate cell metabolism and program the adaptive immune response for clearing infection. IFN actions can also impart cell death, thus limiting virus expansion across tissues. What are the genes that mediate these actions? Recently, genome-wide association studies (GWAS) aimed at defining genes that link with control or susceptibility to SARS-CoV-2 infection have identified IFN response signaling and ISGs as essential factors of innate immune defense against infection, including Ifnar1 (type I IFN receptor subunit), Tyk2 (protein kinase of the Jak-STAT pathway of IFN signaling), and Oas1 (an ISG with known antiviral and immune-modulatory activities). Furthermore, autoantibodies against type I IFNs also strongly associate with severe COVID-19 disease. These studies indicate that IFN and specific ISGs are paramount for defense against SARS-CoV-2 wherein their activities are important for the early control of infection and disease. IFN limits cell to cell, tissue, and systemic spread of virus through autocrine and paracrine actions to induce ISGs and antiviral activity in infected cells and importantly in bystander cells. Beyond a few ISGs we know little of what ISGs control SARSCoV-2 infection nor do we know how IFN impacts infection in the many different tissues implicated in COVID-19 and systemic disease severity. How is it that most people control infection and are asymptomatic or only progress to mild COVID19 and recovery? Research to address this question should focus on understanding IFN and ISG actions in innate immunity to infection. Findings in this area could lead to development of revised or novel therapeutic regimen that direct ISG actions to clear infection and protect against COVID-19.

Michael Gale, Jr. Ram Savan University of Washington School of Medicine Seattle, WA

USA

\footnotetext{
${ }^{1}$ University of Washington School of Medicine, Seattle, Washington, USA.

${ }^{2}$ Icahn School of Medicine at Mount Sinai, New York, New York, USA.

${ }^{3}$ National Cancer Institute, Rockville, Maryland, USA.

${ }^{4}$ National Cancer Institute, Frederick, Maryland, USA.
}

*Correction added on October 20, 2021 after first online publication of May 17, 2021: The fourth author name has been corrected from Ludmila Prokunina-Olsso to Ludmila Prokunina-Olsson. 


\section{Type I/III IFN and SARS-CoV-2: More Research Is Needed}

While SARS-CoV-2 encodes multiple mechanisms that reduce type I/III IFN induction as well as type I/III IFN signaling, pretreatment of cells and animals with type I/III IFN inhibits virus replication. This opens the possibility to use type I/III IFNs for the treatment of SARS-CoV-2 infections. However, one should consider that type I IFN treatment, especially after infection, might increase disease severity due to its pro-inflammatory properties. In this respect, type III IFN, signaling mainly only in epithelial cells due to the tissue and cell type restriction of its receptor, might offer a safer alternative for the therapeutic treatment of SARS-CoV-2 infections. In any case, such a treatment has better chances to be effective given very early after infection or as a prophylactic, especially if administered locally to the respiratory tract. In any case, more research is needed on how to exploit the potent antiviral properties of IFNs for the treatment of viral infections without unwanted local and systemic side effects.

Adolfo Garcia-Sastre Icahn School of Medicine at Mount Sinai New York, NY

USA

\section{One Less Problem with IFN Therapy for COVID-19: It Will Not Induce ACE2 Expression and Increase Infection}

Mitigation of COVID-19 using IFN therapies could be preventive or early post-exposure and possibly topical, restricted to the respiratory tract. In addition to the direct antiviral effect, the goal of this intervention is to prevent progression to more severe disease, such as by preserving ACE2 on the cell surface. In addition to being a SARSCoV-2 receptor, ACE2 is an important factor in the reninangiotensin system and its deficit due to co-internalization with the virus may aggravate disease conditions. However, ACE2 induction, not deficit, was one of the early concerns related to IFN-based therapy for COVID-19. It has been proposed that ACE2 is an interferon-stimulated gene (ISG) induced by both the virus and endogenous or exogenous IFNs. Thus, it was speculated that IFN treatment would induce ACE2 expression and increase viral uptake (Ziegler et al. 2020). We (Onabajo et al. 2020) and two other groups ( $\mathrm{Ng}$ et al. 2020; Blume et al. 2021) showed that a novel truncated transcript, which we designated as delta ACE2 (dACE2) is an ISG induced by IFNs and viruses. Previous analyses were based on measuring the combined expression of ACE2 and dACE2 and thus were misleading. However, dACE2 lacks half of the N-terminal domain necessary for interaction with the viral spike protein and does not appear to bind the virus. Thus, IFN-driven induction of dACE2 was not increasing the viral uptake in vitro. On the other hand, ACE2 acts as a viral receptor but is not an ISG. What does this finding mean for SARS-CoV-2 infection and possible COVID-19 treatment with IFNs? In the absence of ACE2 induction, enhanced viral uptake is one less problem to worry about when considering possible side effects of using IFN-based therapy for COVID-19.

\section{References}

Blume C, et al. 2021. A novel ACE2 isoform is expressed in human respiratory epithelia and is upregulated in response to interferons and RNA respiratory virus infection. Nat Genet 53:205-214.

$\mathrm{Ng} \mathrm{KW}$, et al. 2020. Tissue-specific and interferon-inducible expression of nonfunctional ACE2 through endogenous retroelement co-option. Nat Genet 52:1294-1302.

Onabajo OO, et al. 2020. Interferons and viruses induce a novel truncated ACE2 isoform and not the full-length SARS-CoV-2 receptor. Nat Genet 52:1283-1293.

Ziegler CGK, et al. 2020. SARS-CoV-2 Receptor ACE2 Is an Interferon-Stimulated Gene in Human Airway Epithelial Cells and Is Detected in Specific Cell Subsets across Tissues. Cell 181:1016-1035 e19.

Ludmila Prokunina-Olsson National Cancer Institute Rockville, MD USA

\section{Interferon Therapy for SARS-CoV-2: Is It Underappreciated?}

SARS-CoV-2, a large family of coronaviruses, impacts worldwide population health and it is imperative to design new strategies and treatment options to combat this pandemic. The pathogenicity of SARS-CoV-2 is due to immune evasion that includes inactivation of antiviral and immunomodulatory genes such as interferons at the early stages mediated by viral proteins (Lei et al. 2020; Xia et al. 2020). Despite the fact that type I and type III interferons (IFNs) are used in the treatment of multiple viral infections, it is critical that the outcome of the use of interferons will depend on the study models and the study conditions (Lei et al. 2020; Xia et al. 2020). Accumulating evidence suggests that type I or type III IFNs can reduce SARS$\mathrm{CoV}-2$ infection if used early in infection, thus providing proof of principle that type I or type III IFNs can be utilized in combination therapy as potential antiviral treatments (Vanderheiden et al. 2020; Felgenhauer et al. 2020; Zhou et al. 2020). Along these lines, an open-label randomized controlled clinical trial is now underway for the treatment of SARS-CoV-2 with repurposing the antiviral benefits of the FDA-approved drug Remdesivir along with the immunomodulator interferon 1 beta (NCT04647695). The use of interferon beta is important as some patients have been found to express anti-type 1 interferon antibodies but these antibodies do not neutralize interferon beta (Bastrad et al. 2020). The presence of these autoantibodies also raises the possibility and type III interferons may have a true clinical benefit and initial trials with type III interferons are underway (NCT04534673; NCT04342976; NCT04354259; NCT04388709). However, many questions need to be answered: a) whether the type I and type III IFNs along with other antiviral drugs such as Remdesivir can boost immune responses against all the new variants of SARS-CoV-2; b) whether the delayed interferon responses are indeed a benefit for the host or contribute to a severe cytokine storm; c) can the use of interferon 
enhance immunity to future infections and/or alter the response to the vaccines? and d) would widespread use of inhaled/nasal drop interferons be realistic and be practical for many countries and lessen disease severity in countries where immunization rates are low? Currently, the pilot studies and initial observations suggest that in addition to the newly developed vaccines, interferon therapy may bring much more hope to combat this global public health threat.

\section{References}

Bastrad P, et al. 2020. Autoantibodies against type I IFNs in patients with life-threatening COVID-19. Science 370(6515): eabd4585.

Felgenhauer U, Schoen A, Gad HH, Hartmann R, Schaubmar AR, Failing K, Drosten C, Weber F. 2020. Inhibition of SARS-CoV-2 by type I and type III interferons. J Bio.Chem 295(41):13958-13964.

Lei X, Dong X, Wang W, Xiao X, Tian Z, Wang C, Wang Y, Li L, Ren L, Guo F, Zhao Z, Zhou Z, Xiang Z, Wang J. 2020. Activation and evasion of type $I$ interferon responses by SARS-CoV-2. Nat. Commun. 11:3810.
Vanderheiden A, Ralfs P, Chirkova T, Upadhyay AA, Zimmerman MG, Bedoya S, Aoued H, Tharp GM, Pellegrini KL, Manfrededi C, Sorscher E, Mainou B, Lobby JL, Kohlmeier JE, Lowen AC, Shi P-Y, Menachery VD, Anderson LJ, Grakoui A, Bosinger SE, Suthar MS. 2020. Type I and type III interferons restrict SARS-CoV-2 infection of human airway epithelial cultures. J Virol 94(19): e00985-20.

Xia H, Cao Z, Xie X, Zhang X, Chen JJ Y-C, Wang H, Menachery VD, Rajsbaum R, Shi P-Y. 2020. Evasion of type I interferon by SARS-CoV-2. Cell Reports 33(1):108234.

Zhou Q, Chen V, Shannon CP, Wei X-S, Xiang X, Wang X, Wang Z-H, Tebbutt SJ, Kollmann TR, Fish EN. 2020. Interferon- $\alpha 2 b$ treatment for COVID-19. Front Immunol 15; 11:1061.

Kuppusamy Balamurugan
Howard A. Young National Cancer Institute Frederick, MD

USA 